\title{
Research on Blocking Interference of Electromagnetic Pulse Train to Digital Communication Station
}

\author{
Chao $\mathrm{Qi}^{1,}{ }^{*}$, Guanghui Wei ${ }^{1}$, Xiaodong $\mathrm{Pan}^{1}$, Wei $\mathrm{Li}^{2 \text {, a }}$ \\ ${ }^{1}$ National Key Laboratory on Electromagnetic Environment Effects, Shijiazhuang, China \\ ${ }^{2}$ PLA National Defense University Joint Operations College, Shijiazhuang, China

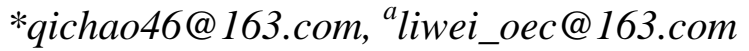

Keywords: electromagnetic pulse train; repetition rate; digital communication radio; BER

\begin{abstract}
In order to study the blocking effect mechanism of electromagnetic pulse string on digital communication radio station, a certain type of digital communication station is used as the test object. The variation of the BER (bit error rate) of the tested station with the amplitude and repetition rate of the electromagnetic pulse train is studied by the electromagnetic pulse injection test. The results show that when the repetition rate is below 50pps, the BER of the tested station increases with the increase of the pulse amplitude, and becomes stable after reaching the criterion of sensitivity criterion, and there is a linear relationship between the repetition rate of the electromagnetic pulse and the error rate of the tested radio station. The higher the repetition rate of the electromagnetic pulse, the greater the BER of the tested radio station. All the tests under existing conditions in the laboratory meet the above conclusions.
\end{abstract}

\section{Introduction}

The electromagnetic environment is becoming more and more complex, and the electronic equipment and system are facing various natural and artificial electromagnetic harassment [1]. It is of great significance to study the mechanism of various interference sources on the electronic equipment in the fields of national defense, space and electronics. Electromagnetic pulse is a part of the battlefield electromagnetic environment. Electrostatic discharge, lightning, nuclear electromagnetic pulse and UWB electromagnetic pulse can all disturb the sensitive equipment. The UWB electromagnetic pulse covers a wide range of spectrum and has high instantaneous electric field intensity. It can be coupled through multiple channels such as antenna and cable, especially the completely exposed antenna, which can form strong impulse voltage and current, cause the equipment work disorder or component damage, and make it lose normal working performance [2]. As an indispensable part of the modern war command system, digital communication equipment is more sensitive to the electromagnetic environment and needs to be studied in depth for its antiinterference performance [3].

In recent years, the research of electromagnetic pulse effect has been paid great attention both at home and abroad. It mainly focuses on the simulation of electromagnetic pulse field, the effect experiment and mechanism analysis of the electromagnetic pulse on the sensitive circuits such as 
single-chip microcomputer and radio fuse, etc. The effect experiment on the communication radio is concentrated on the dead machine and restart under the single pulse. However, there are few studies on blocking effects of electromagnetic pulse trains on digital communication radio. Therefore, this article selects a certain type of digital communication radio as the test equipment. According to the characteristics of the electromagnetic pulse train, the variation regularity of the BER parameters of the communication stations under different pulse repetition rates and different amplitude electromagnetic pulse trains is studied, and the test results are analyzed and summarized.

\section{Test Method}

In the battlefield electromagnetic environment, electrostatic electromagnetic pulses and lightning electromagnetic pulses and nuclear electromagnetic pulses are difficult to form a stable continuous pulse train, and UWB electromagnetic pulses can be achieved. The repetition rate can be as high as 1000 pps or more. Therefore, a high-frequency electromagnetic noise generator is used to simulate an UWB signal and injected into the receiver input terminal of the test equipment through a coupling module for testing. Research shows that the main coupling channel for blocking interference of communication stations is the receiving antenna. Therefore, the injection method is used instead of the irradiation method to perform the test. The results of the injection method and the irradiation method are consistent [4], and the injection method is not easily disturbed by the outside, and the test results have better repeatability.

The transmitting station is connected to a $40 \mathrm{~dB}$ attenuator to simulate long-distance communication. Both the transmitting antenna and the receiving station are placed in a shielded room. The signal generated by the high-frequency electromagnetic noise generator is attenuated by $60 \mathrm{~dB}$ and mixed with the useful signal received by the antenna into the RF front-end receiving channel of the communication station. The specific test configuration is shown in Fig. 1.

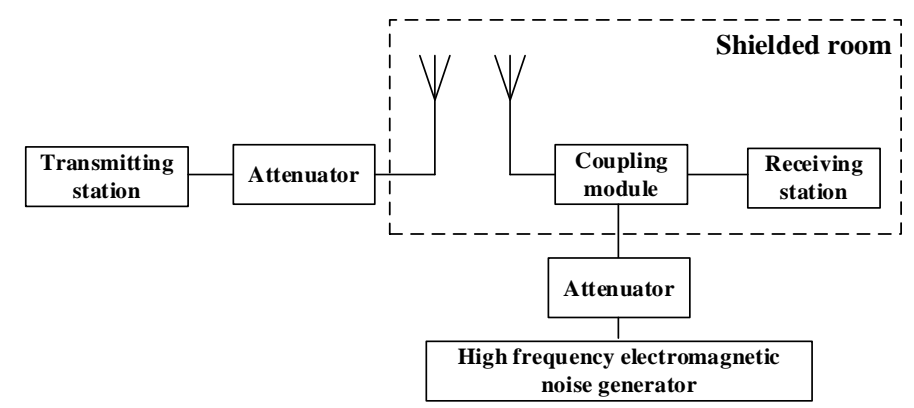

Fig 1 Injection station test configuration diagram

The injection coupling module's specifications are in accordance with the differential mode injection method. The frequency range is $100 \mathrm{kHz}$ to $1.5 \mathrm{GHz}$, the frequency response flatness is better than $2 \mathrm{~dB}$, the injection coupling degree is $10 \mathrm{~dB}$, and the injection and output ports have no phase mutation. The interference signal injected into the input terminal of the test station is still a rectangular pulse train.

The test station is a type of digital communication station. The BER criterion is selected when evaluating the interference effect of digital communication using binary random sequences and digital voice sources. Therefore, the transmission bit error rate of digital communication stations is the observation index. Through previous research, it has been found that the BER values of the tested stations under different repetition rate electromagnetic pulses are quite different. It is impossible to use a fixed standard to measure whether the station is in a disturbed state. After entering a large number of experimental analysis studies, choose the test point with the highest BER under different repetition rates and tending to a stable value as the sensitive point. The BER of this point is defined 
as the sensitivity BER, and the injection voltage when the sensitive BER reached is defined as the sensitive voltage.

The amplitude and interval time of the output signal of the high-frequency electromagnetic noise generator is changed when the normal working state of the radio station. The value of the station BER is measured when the injected signal is injected, and the influence of the injected signal on the digital communication station is judged by the size of the BER.

\section{Test Results}

With low pulse repetition rate, the BER of the digital communication station is very unstable with low injection voltage. When the injection voltage is increased, the interference effect is obviously enhanced and tends to be stable, and the BER no longer changes after reaching the sensitive BER. With high pulse repetition rate, the experimental results are more stable. Due to the limited error rate measurement method, the BER increases step by step. When the sensitive BER is reached, the BER does not change. Some test results are shown in Fig 2. It can be seen that the amplitude of the pulse voltage directly affects the interference effect, but there is an upper limit and cannot be increased without limitation for blocking interference at the same pulse repetition rate.

The upper limit of the sensitivity error rate under different pulse repetition rates is quite different. When the repetition rate of the electromagnetic pulse is high, the BER of the communication station is also larger. Some of the test results are shown in Fig. 3. In the known experimental data, the BER of the communication station is linearly positively correlated with the pulse repetition rate.
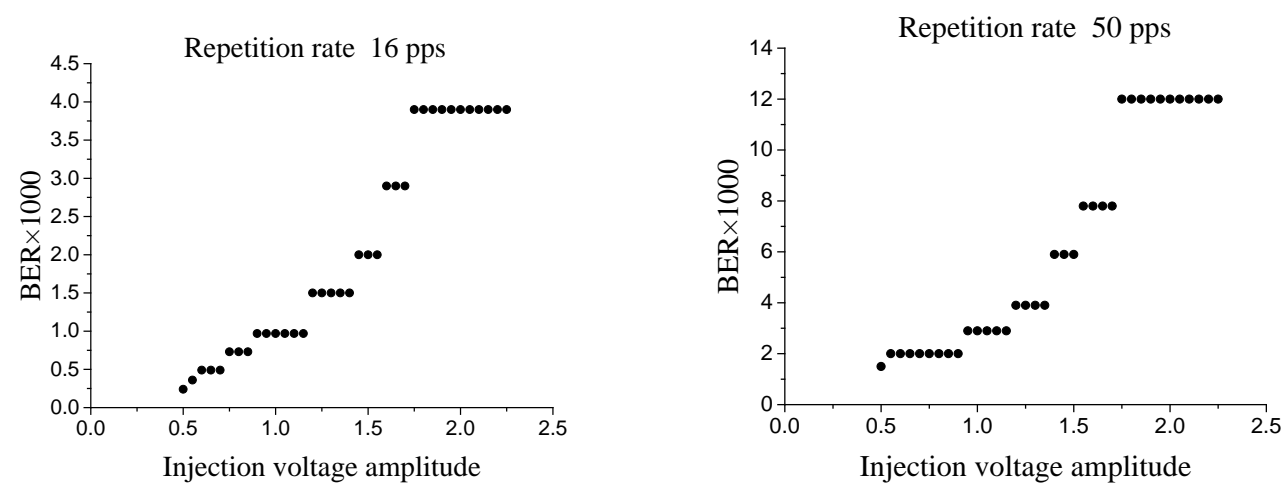

Fig 2 BER with injection voltage at different pulse repetition rates

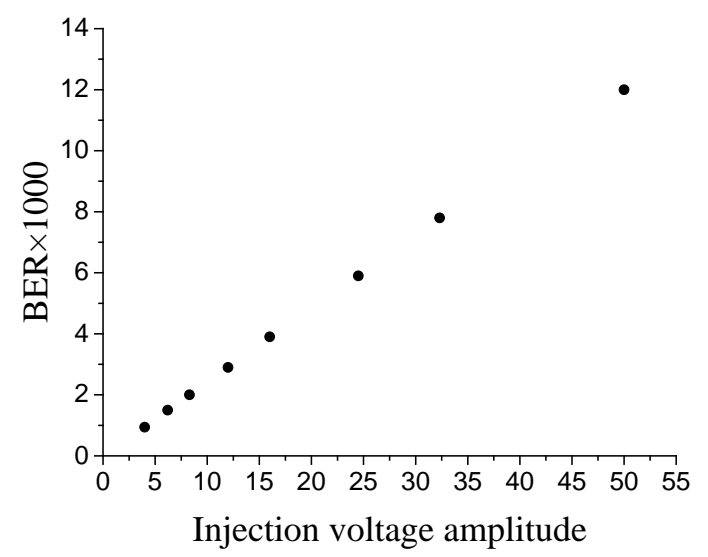

Fig 3 Sensitive BER at different pulse repetition rates 


\section{Conclusion}

Electromagnetic pulses can cause blocking interference to digital communication stations. The amplitude and repetition rate of electromagnetic pulses are all direct factors that affect the degree of interference. The importance of different parameters to interference is quite different. Increasing the amplitude of the electromagnetic pulse can exacerbate the blocking interference, but there is a certain upper limit, which cannot be increased without limit, and can be effectively proved under the existing experimental conditions. The blocking degree of the digital communication station will be directly affected by the repetition rate of the electromagnetic pulse, and it shows a significant linear increase relationship. In laboratory conditions, the maximum pulse repetition rate also satisfies the linear condition. Through the relational prediction, the linear relationship will be maintained at about 400 pps.

\section{References}

[1] Liu S H, Wu Z C, Zhang X J. Electromagnetic environment effect and its development trends[J]. National Defense Science and Technology,2008,29(1).1-6.

[2] Wei G, Geng L, Pan X. Effects of Electromagnetic Radiation on Communication Stations [J]. High Voltage Engineering, 2014,40 (09): 2685-2692.

[3] Li W, Wei G, Pan X, Lu X, Wan H. Study on Electromagnetic Susceptibility Criterion of Typical Communication Equipment [J]. Journal of Microwaves, 2016,32 (06):70-75.

[4] Lu X, Wei G, Pan X, et al. A Pulsed Differential-Mode Current Injection Method for Electromagnetic Pulse Field Susceptibility Assessment of Antenna Systems [J].IEEE Transactions on Electromagnetic Compatibility, 2015, 57(6):1435-144 